\title{
Pediatric Psychiatric Disorder
}

National Cancer Institute

\section{Source}

National Cancer Institute. Pediatric Psychiatric Disorder. NCI Thesaurus. Code C92190.

A category of psychiatric disorders which includes disorders most commonly identified in infancy, childhood, or adolescence. 